\title{
Complete Occlusion of Bilateral Internal Carotid Artery in a Marijuana Smoker: A Case Report
}

\author{
Dikshya Sharma $^{\mathrm{a}, \mathrm{c}}$, Udip Dahal ${ }^{\mathrm{a}}$, Edward Yu $^{\mathrm{b}}$
}

\begin{abstract}
Here we described a case of a young male who developed stroke attributable to heavy marijuana smoking. Although, instances of stroke due to cannabis smoking have been reported earlier, this case is distinctive due to the fact that the patient had bilateral complete occlusion of internal carotid artery (ICA) at a very early age. In view of studies that have correlated marijuana smoking to intracranial stenosis and atherogenesis, this case of bilateral ICA occlusion illustrates the severity and burden of the disease.
\end{abstract}

Keywords: Internal carotid artery; Atherogenesis; Marijuana

\section{Introduction}

Cannabis smoking has been surrounded by number of controversies. With legalization of marijuana in a number of states in US, its use, both for medical or recreational has been in increasing. There have been a number of researches exploring its pros and cons, and it is important to identify health conditions related to it. With this case report we present an unusual case of stroke due to occlusion of bilateral carotids with marijuana inhalation.

\section{Case Report}

A 37-year-old male with no significant medical history was brought in by his father with complaints of worsening mental status and functional decline for a couple of months. According to the father, his son had been behaving strangely, and was socially inept, indifferent to his surrounding, lacked attention, did not participate in conversation and was unsteady. On ex-

Manuscript submitted July 13, 2017, accepted July 31, 2017

aStaten Island University Hospital, 475 Seaview Avenue, Staten Island, NY 10305, USA

b Department of Neurology, Staten Island University Hospital, Staten Island, NY 10305, USA

${ }^{\mathrm{c} C o r r e s p o n d i n g ~ A u t h o r: ~ D i k s h y a ~ S h a r m a, ~ S t a t e n ~ I s l a n d ~ U n i v e r s i t y ~ H o s p i t a l, ~}$ 475 Seaview Avenue, Staten Island, NY 10305, USA.

Email: dikshya_sh896@yahoo.com

doi: https://doi.org/10.14740/jocmr3132w amination, the patient appeared alert, awake and oriented but disheveled and lack of attention. Motor, sensory and cranial nerves were grossly intact except for slight weakness of left arm and leg. With inertia of the left hand on nose to finger test, he had unsteady gait with decreased postural control. He reported to have been smoking about $3 \mathrm{~g}$ of marijuana since the age of 12 every day and also had a history of cigarette smoking.

His vitals including initial lab values were all normal. A urine drug screen was positive for cannabinoids. Computed tomography (CT) scan of head showed mild parenchymal volume loss and prior chronic ischemic changes in the periventricular region. Subsequently, magnetic resonance imaging MRI of head was done (Fig. 1a-c) that revealed multiple foci of abnormal signal intensity in the right cerebral hemisphere and a small focus in the left parietal region. There was absence of blood flow in the region of the internal carotid artery (ICA) bilaterally. The possibility of occlusion and/or dissection of ICA were considered and CT angiogram of the head and neck was recommended for further evaluation.

CT scan angiogram of head and neck (Figs. 2, 3) showed complete occlusion of ICA bilaterally. The patient was transferred to stroke unit and was started with dual antiplatelets aspirin and Plavix. Although overall status remained the same, his mental lucidity gradually started to improve. Extensive workup was done to find the cause of the ICA obstruction. He had normal lipid profile, hypercoagulability workup and vasculitis panel. Screening for human immunodeficiency virus (HIV), heavy metals, Wilsons and hematochromosis showed negative results and no atrial fibrillation was detected on telemetry. He further underwent transesophageal echocardiography (TEE) which revealed no cardiac clots.

He was diagnosed to have acute and chronic ischemic stroke due to gradually progressing thrombotic occlusion of ICA bilaterally secondary to atherogenesis. After 8 days of hospitalization, with joint decision of neurology and vascular he was started on anticoagulation with heparin drip as a bridge to coumadin. After he attained the international normalized ratio (INR) of 2 he was discharged to inpatient rehabilitation unit.

\section{Discussion}

Cannabis is the most used illicit drug worldwide. About $2.5 \%$ of the world population consumes cannabis; and its use has grown more rapidly in developed regions like North America, 

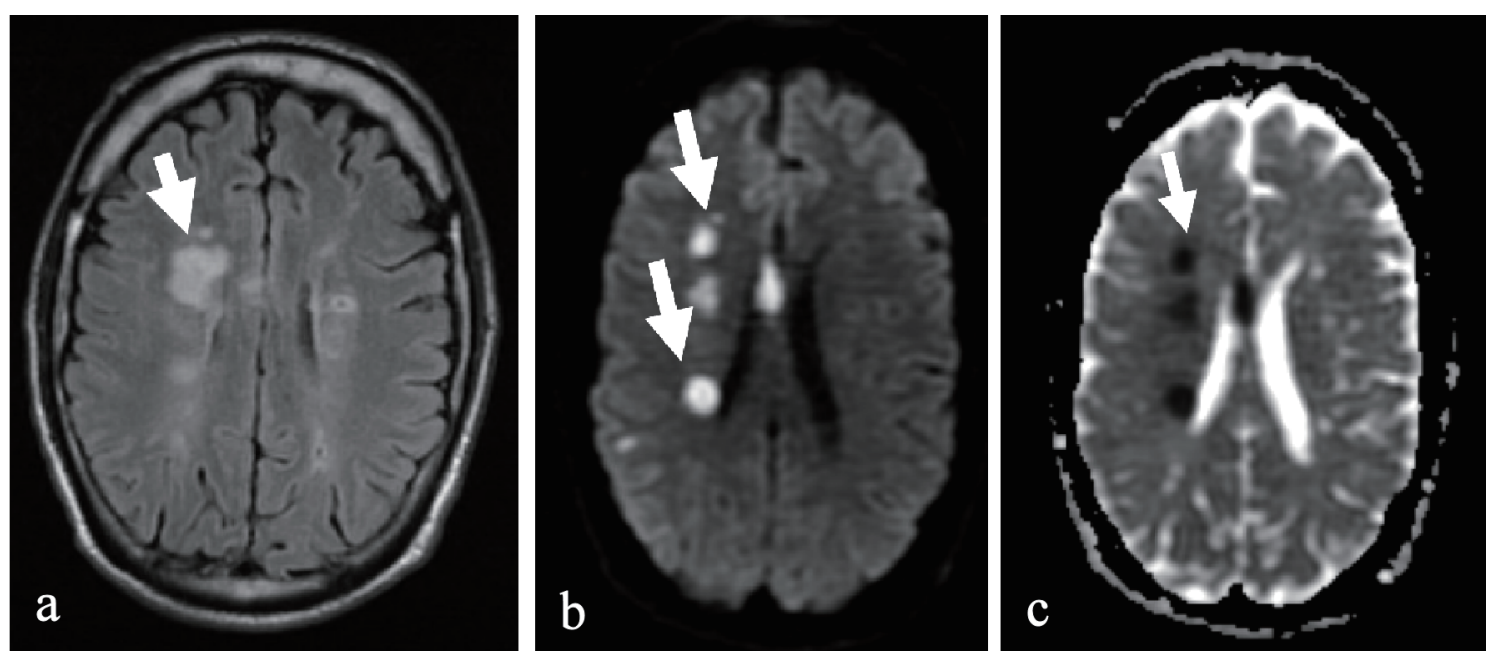

Figure 1. MRI head showed multiple foci of abnormal signal intensity in the right cerebral hemisphere and a small focus in the left parietal region representing acute ischemic changes as shown by arrow. (a) T2 flair. (b) Diffusion weighted image. (c) Apparent diffusion coefficient.

Western Europe and Australia. The major psychoactive constituent tetrahydrocannabinol has been shown to have temporal associations to serious adverse events as acute myocardial infarction [1], cardiomyopathy, sudden cardiac death [2-4] effects on brain development, various acute neurological deficits [5-8] and stroke.

Most of these cases of stroke or acute neurological dysfunction were described in male who smoked marijuana heavily (14 cigars/day or about $5-7 \mathrm{~g}$ of marijuana) for more than a decade. Some cases occurred while the drug was actually being smoked and some reports illustrated recurrence of stroke in patients who didn't comply with cessation. Potential mecha- nism behind many of these effects is attributable to marijuanainduced hypertension, dyslipidemia, higher caloric intake [9] and increased platelet coagulability [10].

About 2-39\% of cases of ischemic stroke in young adults are suspected to be associated with recreational drug use. Cases of stroke after marijuana smoking have been reported in many prior studies where its use was found to be a prominent risk factor [11-13]. Most of the cases described were acute neurological dysfunction during actual marijuana inhalation suggesting a reversible effect of cannabis inhalation on cranial circulation likely due to spasm or, increase in blood pressure $[14,15]$. Later studies showed that misuse of marijuana was

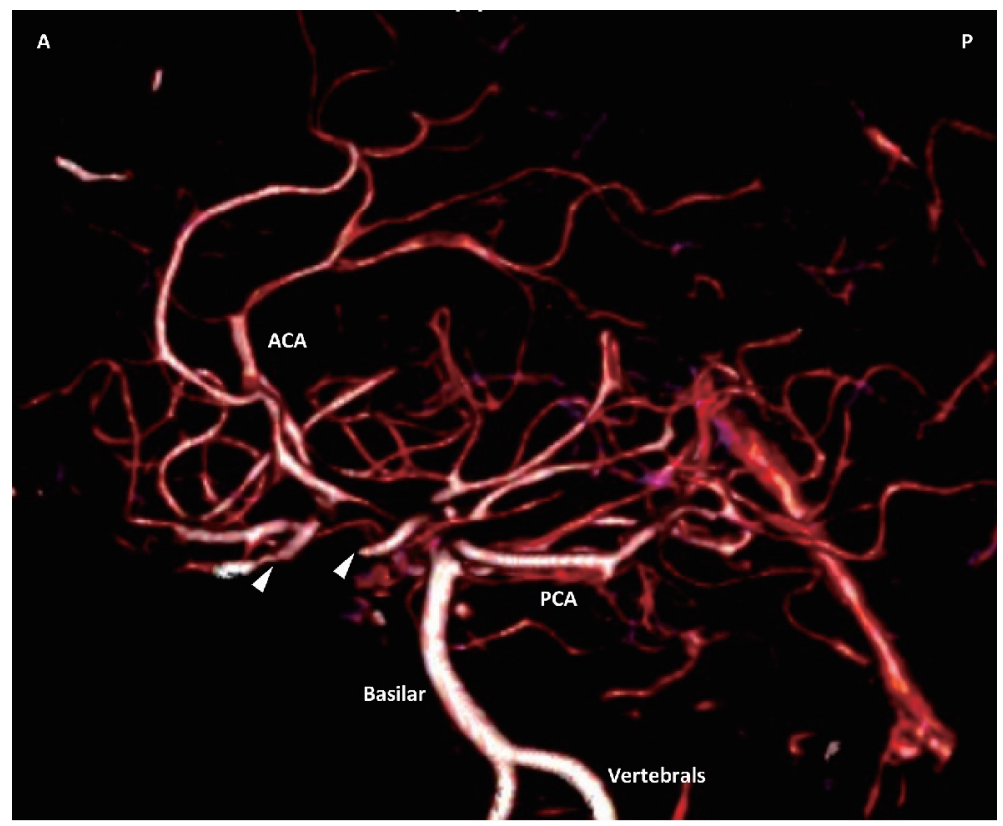

Figure 2. CT angiography of the head and neck with $125 \mathrm{~mL}$ Optiray 350 contrast enhanced 3D volume rendered image, with white arrowheads showing ICA occlusions. 


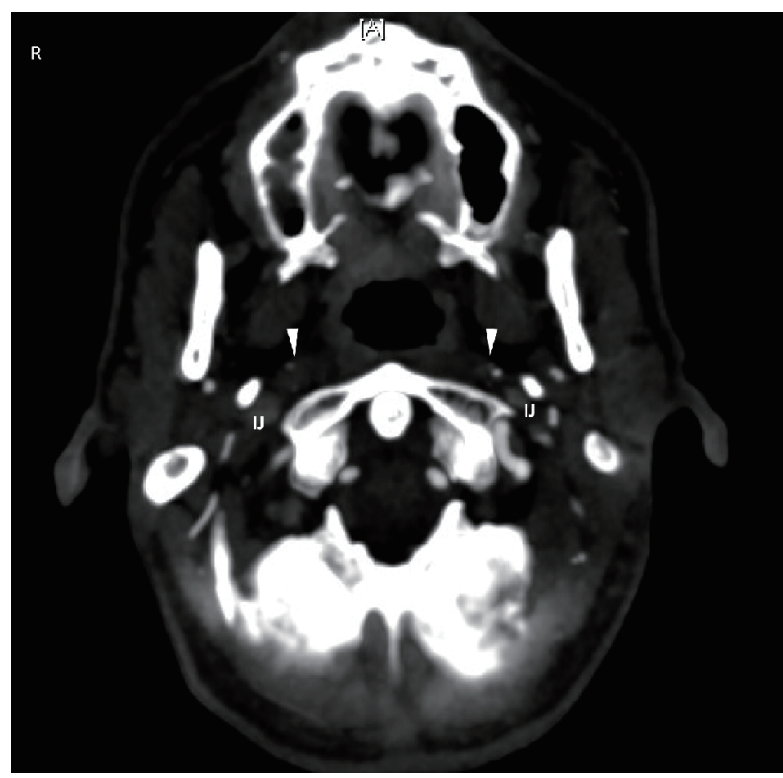

Figure 3. CT angiography of the head with $125 \mathrm{~mL}$ Optiray 350 contrast, with white arrowheads showing the ICA occlusions with calcification. IJ: internal jugular.

more frequently associated with stroke in young population with no other traditional risk factors. A prospective cohort on 48 young patients also revealed significant association between multifocal intracranial stenosis and marijuana consumption with odds ratio of $113(9-5,047)$ and $\mathrm{P}$ value $<0.001$ [16]. Similarly, a case series by Singh et al [13] described a causal role of cannabis in cases of ischemic stroke by showing recurrence of symptoms in a few patients with re-exposure. Majority of the patients in mentioned studies had posterior circulation strokes. Recently a France-based cohort study found that majority strokes in marijuana abusers were caused by intracranial arterial stenosis [17]. However, none of the studies described above would explain our case of ischemic stroke secondary to bilateral thrombotic athero-occlusive disease of ICA. Relation between marijuana smoking and atherosclerotic coronary heart disease has been studied before. Animal model studies showed that cannabinoids can potentially lead toward progression of atherosclerosis by its proatherogenic effect on endothelial cells, vascular smooth muscle cells and lipid metabolism precipitating acute coronary syndrome and decreasing angina threshold [18]. Same process could explain thrombosis of ICA in our case leading to ischemic stroke. Such case of bilateral occlusion secondary to chronic marijuana smoking has been never mentioned in literatures. Although in this case a direct correlation between marijuana and carotid occlusion cannot be established due to presence of confounding factors, we can argue that it might have contributed to the progression of atherosclerosis. However, definitive conclusions as to the underlying pathophysiology and hence exact mechanism is still not well established and hence further research in this arena should be sought. Fighting stroke must remain a priority in young patients and hence potential occurrence of stroke associated with cannabis should be reported more for better awareness in the society. Also community should focus to in- tensify research regarding the safety of marijuana use as cannabis is not as harmless as was thought, and cerebrovascular risk is probably underestimated.

\section{Acknowledgments}

Non applicable.

\section{Conflict of Interest}

The authors declare that they have no competing interests.

\section{Author Contributions}

Dikshya sharma made substantial contributions to conception and design, or acquisition of data, or analysis and interpretation of data. Udip Dahal was involved in drafting and revising the manuscript. Edward Yu made the final approval of the version to be published.

\section{References}

1. Mittleman MA, Lewis RA, Maclure M, Sherwood JB, Muller JE. Triggering myocardial infarction by marijuana. Circulation. 2001;103(23):2805-2809.

2. Ting JY. Reversible cardiomyopathy associated with acute inhaled marijuana use in a young adult. Clin Toxicol (Phila). 2007;45(4):432-434.

3. Kaushik M, Alla VM, Madan R, Arouni AJ, Mohiuddin SM. Recurrent stress cardiomyopathy with variable regional involvement: insights into etiopathogenetic mechanisms. Circulation. 2011;124(22):e556-557.

4. Bachs L, Morland H. Acute cardiovascular fatalities following cannabis use. Forensic Sci Int. 2001;124(23):200-203.

5. Garrett CP, Braithwaite RA, Teale JD. Unusual case of tetrahydrocannabinol intoxication confirmed by radioimmunoassay. Br Med J. 1977;2(6080):166.

6. Mohan H, Sood GC. Conjugate Deviation of the Eyes after Cannabis Indica Intoxication. Br J Ophthalmol. 1964;48:160-161.

7. Cooles P, Michaud R. Stroke after heavy cannabis smoking. Postgrad Med J. 1987;63(740):511.

8. Zachariah SB. Stroke after heavy marijuana smoking. Stroke. 1991;22(3):406-409.

9. Rodondi N, Pletcher MJ, Liu K, Hulley SB, Sidney S, Coronary Artery Risk Development in Young Adults $\mathrm{S}$. Marijuana use, diet, body mass index, and cardiovascular risk factors (from the CARDIA study). Am J Cardiol. 2006;98(4):478-484.

10. Dahdouh Z, Roule V, Lognone T, Sabatier R, Grollier G. Cannabis and coronary thrombosis: What is the role of platelets? Platelets. 2012;23(3):243-245.

11. Phillips MC, Leyden JM, et al. Ischemic stroke among 
young people ages 15 to 50 years in Adelaide. South Australia Med J Aust. 2011;195:610-614.

12. Geller T, Loftis L, Brink DS. Cerebellar infarction in adolescent males associated with acute marijuana use. Pediatrics. 2004;113(4):e365-370.

13. Singh NN, Pan Y, Muengtaweeponsa S, Geller TJ, CruzFlores S. Cannabis-related stroke: case series and review of literature. J Stroke Cerebrovasc Dis. 2012;21(7):555560 .

14. Barber PA, Pridmore HM, Krishnamurthy V, Roberts S, Spriggs DA, Carter KN, Anderson NE. Cannabis, ischemic stroke, and transient ischemic attack: a case-control study. Stroke. 2013;44(8):2327-2329.

15. Marinella MA. Stroke after marijuana smoking in a teenager with factor $\mathrm{V}$ Leiden mutation. South Med J. 2001;94(12):1217-1218.

16. Wolff V, Lauer V, Rouyer O, Sellal F, Meyer N, Raul JS, Sabourdy C, et al. Cannabis use, ischemic stroke, and multifocal intracranial vasoconstriction: a prospective study in 48 consecutive young patients. Stroke. 2011;42(6):1778-1780.

17. Wolff V, Zinchenko I, Quenardelle V, Rouyer O, Geny B. Characteristics and prognosis of ischemic stroke in young cannabis users compared with non-cannabis users. J Am Coll Cardiol. 2015;66(18):2052-2053.

18. Singla S, Sachdeva R, Mehta JL. Cannabinoids and atherosclerotic coronary heart disease. Clin Cardiol. 2012;35(6):329-335. 